\title{
Usefulness of consecutive C-reactive protein measurements in follow-up of severe community-acquired pneumonia
}

\author{
A.H.W. Bruns*, J.J. Oosterheert*, E. Hak, ${ }^{\#,}$ and A.I.M. Hoepelman*,+
}

ABSTRACT: Despite the introduction of new inflammatory markers, C-reactive protein (CRP) remains commonly used in patients hospitalised with severe infections. However, evidence on the usefulness of consecutive CRP measurements is still unclear. The clinical relevance of consecutive CRP measurements was studied in follow-up of antibiotic treatment in patients with severe community-acquired pneumonia (CAP).

In a prospective multicentre trial, CRP levels were measured on admission, and on days 3 and 7. Patients were followed clinically for 28 days.

Aetiology could be determined in 137 (47.4\%) out of the 289 patients included. In 122 (38.8\%) patients, initial antibiotic therapy was appropriate. A decline of $<60 \%$ in CRP levels in 3 days and a decline of $<\mathbf{9 0 \%}$ in CRP levels in $\mathbf{7}$ days were both associated with an increased risk of having recieved inapproriate empiric antibiotic treatment (day 0-3, odds ratio (OR) 6.98, 95\% confidence interval (CI) 1.56-31.33 and day 0-7, OR 3.74, 95\% $\mathrm{Cl} 1.12-13.77$ ).

In conclusion, consecutive C-reactive protein measurements are useful in the first week in follow-up of antibiotic treatment for severe community-acquired pneumonia when taking the causative microorganism and use of steroids into account. A delayed normalisation of C-reactive protein levels is associated with a higher risk of having received inappropriate antibiotic treatment.

KEYWORDS: Antibiotic treatment, community-acquired pneumonia, C-reactive protein, follow-up

ommunity-acquired pneumonia (CAP) is the major cause of death due to infectious diseases in the western world and accounts for an increasing figure of $\geqslant 20$ admissions per 1,000 inhabitants annually [1]. Current guidelines advise combination therapy with $\beta$ lactam and macrolide antibiotics for initial treatment of severe CAP $[2,3]$. Consequently, management of severe CAP accounts for high utilisation of healthcare resources and antibiotic consumption, leading to a risk of emerging resistance. In the USA, annual estimated costs for treating CAP exceed US\$12 billon and in several countries an increase in macrolide-resistant strains has been observed [4, 5].

Once aetiology of CAP has been established, pathogen-directed antibiotic therapy can be initiated and a test indicative of aetiology early in the course of disease would be a worthwhile target for the reduction of antibiotic consumption. Unfortunately, thus far no biomarker has been found to have sufficient sensitivity and specificity to guide initial therapy, and protocols for guidance of empirical antibiotic treatment must be relied on $[2,3]$. However, an alert for an unfavourable response to treatment early in follow-up, as an increased inflammatory response, suboptimal drug levels, or inappropriate empirical treatment, could help in optimising treatment for CAP patients. Before aetiology has been established, or when aetiology cannot be established, an indicator of the appropriateness of empirical antibiotic therapy may contribute to a more tailored approach in antibiotic treatment early in the course of the disease. Furthermore, such an indicator might help in continuing tailored antibiotic therapy, determining the length of antimicrobial treatment, and guiding a switch from intravenous to oral antibiotic therapy [6]. Hypothetically, these strategies may contribute to a reduction in antibiotic consumption.

The determination of the serum concentration of C-reactive protein (CRP) is a rapid, simple and inexpensive procedure and consecutive CRP measurements have become routine clinical practice in the follow-up of patients hospitalised

\section{AFFILIATIONS}

*Division of Medicine, Dept of Internal Medicine and Infectious Diseases,

\# Julius Center for Health Sciences and Primary Care,

"Paediatric Immunology, Wilhelmina Children's Hospital, and +Eijkman-Winkler Institute for Microbiology, Infectious Diseases and Inflammation, University Medical Center Utrecht, Utrecht, The Netherlands.

\section{CORRESPONDENCE}

A.I.M. Hoepelman

Dept of Internal Medicine and Infectious Diseases University Medical Center Utrecht Room F02.126

P0 Box 85500

3508 GA Utrecht

The Netherlands

Fax: 31302523741

E-mail: I.M.Hoepelman@

umcutrecht.nl

Received:

January 082008

Accepted after revision:

May 092008

STATEMENT OF INTEREST

None declared.

European Respiratory Journal Print ISSN 0903-1936

Online ISSN 1399-3003 
with severe infections [7]. However, despite its frequent use, evidence on the usefulness of consecutive CRP measurements for follow-up of antibiotic treatment for severe CAP is lacking. Few studies have addressed CRP kinetics in the follow-up of CAP previously, and these are on a relatively small scale and have not taken aetiology into account $[8,9]$. A recent study has pointed out that high serum levels of CRP, interleukin (IL)-6 or, procalcitonin (PCT) are associated with a higher risk of any treatment failure [10]. However, the introduction of newer inflammatory markers, such as PCT, IL-6 and neopterin emphasises the need to clarify the position of the older and less costly markers, such as CRP [11]. To determine the clinical relevance of consecutive CRP measurements in follow-up of antibiotic treatment in patients with severe CAP, the present study examined the predictive value of delayed normalisation of CRP levels for the risk of having received inappropriate empirical antibiotic therapy or developing an unfavourable outcome.

\section{MATERIALS AND METHODS Setting and study population}

The current study is a retrospective analysis of data derived from a multicentre, prospective randomised controlled trial on the cost effectiveness of an early switch from i.v. to oral antibiotic therapy for severe CAP [12]. The trial was conducted in five teaching hospitals (Meander Medical Centre (Amsterfoort), Diakonessen Hospital (Utrecht), Rijnstate Hospital (Arnhem), St Antonius Hospital (Nieuwegein) and Jeroen Bosch Hospital (s-Hertogenbosch)) and two university medical centres (Academic Medical Centre Amsterdam (Amsterdam) and University Medical Centre Utrecht (Utrecht)) in the Netherlands from July 2000 to June 2003. All adult patients (age $\geqslant 18 \mathrm{yrs}$ ) admitted to one of the participating hospitals due to CAP were eligible for inclusion. CAP was defined as present in cases with at least two symptoms of acute lower respiratory tract infection with onset before hospital admission and a new or progressive pulmonary infiltrate on chest radiograph. Severe CAP was defined as a Pneumonia Severity Index (PSI) score $>90$ or according to the American Thoracic Society definitions [13, 14]. All patients gave written informed consent prior to enrolment and the study was approved by the medical ethics committees of all participating hospitals. Patients with interstitial pneumonia, cystic fibrosis, a history of colonisation with Gram-negative bacteria due to structural damage to the respiratory tract, a life expectancy of $<1$ month because of an underling disease, severe neutropenia $\left(<0.5 \times 10^{9}\right.$ neutrophils $\left.\cdot \mathrm{L}^{-1}\right)$ or HIV infection with a CD4 count $<200$ cells $\cdot \mathrm{mm}^{-3}$, infections other than pneumonia necessitating treatment with i.v. antibiotics, and patients admitted directly to an intensive care unit (ICU) were excluded.

\section{Data collection and CRP assay}

On admission, demographic data and clinical signs and symptoms were recorded. Severity of disease was determined by PSI score and Acute Physiology and Chronic Health Evaluation (APACHE) II score [14, 15]. Laboratory tests, microbiological tests and a chest radiograph were obtained before empirical antibiotic treatment was instituted. Patients were followed for a maximum of 28 days. Serum samples to quantify the serum CRP concentration were obtained on admission in the emergency department and on days 3 and 7 of hospitalisation. Serum concentrations of CRP were measured by monoclonal immunoassay using a VITROS analyser (Ortho-Clinical Diagnostics, Johnson and Johnson, Amersham, UK) The normal reference range for this assay is $<10 \mathrm{mg} \cdot \mathrm{L}^{-1}$.

\section{Microbiological evaluation}

Sputum samples (when available) and blood samples were collected, cultured and evaluated according to standard procedures [12]. Sputum samples were considered adequate and subsequently cultured if $\geqslant 25$ polymorphonuclear neutrophils and $<10$ epithelial cells were present in each highpower field. Urinary antigen tests (Binax Inc., Portland, ME, USA) were used to detect antigens of Streptococcus pneumoniae and Legionella pneumophila. Acute and convalescent sera were collected and tested for Mycoplasma pneumoniae, L. pneumophila and Chlamydia pneumoniae. The following results were considered indicative of infection: for M. pneumoniae, a four-fold or greater increase in titre in paired sera or a single titre of 1:40 or greater (immune fluorescence agglutination, Serodia-MycoII; Fujirebio, Malvern, PA, USA) [16]; for L. pneumophila, a fourfold increase in the antibody titre to 1:128 or greater, or single titres of 1:256 or more [17]; and for C. pneumoniae, detection of immunoglobulin (Ig)M above established values, seroconversion of IgG between acute and convalescence samples, high amounts of IgG in single titres, or a combination of these (ELISA; Savyon Diagnostics, Ashdad, Israel). Pathogenic microorganisms cultured from blood or sputum, detected by urinary antigen test or a seroconversion were considered the cause of the episode of CAP.

\section{Definitions}

Appropriate antibiotic treatment was defined as at least one antibiotic covering all of the causative pathogens identified, as determined by the sensitivity pattern in the antibiogram. Guidelines of the Dutch antimicrobial committee (SWAB) were used to determine the appropriateness of antibiotic therapy for each aetiology [18].

Early treatment failure was defined as clinical instability (respiratory rate $>25$ breaths $\cdot \mathrm{min}^{-1}$; oxygen saturation $<90 \%$ as measured by pulse oximetry; $\mathrm{Pa}_{1} \mathrm{O}_{2}<7.3 \mathrm{kPa}(<55 \mathrm{mmHg})$; haemodynamic instability or acute alterations in mental state), ICU admission or mortality in the first 3 days of admission [19]. Late treatment failure was defined as clinical deterioration or complications including mortality, the need for mechanical ventilation, readministration of i.v. antibiotics after a switch to oral therapy, readmission for pulmonary infection after discharge, or an increase in body temperature after initial improvement in the follow-up period [20]. The per cent decline in CRP levels reflects the relative changes in CRP concentrations in the course of time, calculated in relation to the day 0 CRP concentrations. Delayed normalisation of CRP was defined as a decline of $<60 \%$ in CRP levels in 3 days and a decline of $<90 \%$ in CRP levels in 7 days.

\section{Analytical approach}

In order to investigate the clinical relevance of consecutive CRP measurements in the follow-up of antibiotic treatment for severe CAP, the relationships between baseline CRP levels and patients' demographics, comorbidity, medication use and 
aetiology were initially explored. Subsequently, among patients with established aetiology, the association between the decline in CRP levels and appropriateness of empirical antibiotic treatment was studied. Furthermore, the predictive value of a delayed normalisation of CRP for the risk of having received inappropriate antibiotic treatment or an unfavourable clinical outcome, such as mortality, early treatment failure and late treatment failure, was studied by means of multivariable models.

\section{Statistical methods}

Continuous variables were tested by Mann-Whitney U-tests or paired t-test, where appropriate, and categorical variables were compared using the Chi-squared test. ANOVA was used for comparisons between more than two groups. The rate of decline in CRP levels was dichotomised: the cut-off values of a $60 \%$ decline on day 3 and a $90 \%$ decline on day 7 were determined in line with previous published data and the 75th percentiles of CRP levels on day 3 and 7, after rounding [8]. The association of a delayed decline in CRP levels and the appropriateness of initial antibiotic treatment and clinical outcome was compared by estimation of the odds ratio (OR) with corresponding 95\% confidence intervals (CIs). Correction for patient characteristics, pneumonia severity, and symptoms and signs of pneumonia on admission was performed by multivariate assessment. A p-value of $<0.10$ in univariable analysis or any clinically relevant parameter was used as an entry criterion for multivariate analysis. A p-value of $<0.05$ was considered statistically significant.

\section{RESULTS}

\section{Baseline characteristics}

A total of 289 patients with severe CAP were enrolled in the trial. The patients' mean \pm SD age was $69.7 \pm 13.8$ yrs. The mean PSI and APACHE II scores in the study population were $112.9 \pm 25.7$ and $13.8 \pm 4.6$, respectively. Of the study patients, $180(62.3 \%)$ had a risk-elevating medical condition congestive heart failure, neoplasm, cerebrovascular disease, chronic renal failure, liver disease or chronic obstructive pulmonary disease. Overall, the median serum CRP concentration on admission was $174 \mathrm{mg} \cdot \mathrm{L}^{-1}$ (interquartile range $147-390 \mathrm{mg} \cdot \mathrm{L}^{-1}$; table 1 ). Slightly lower baseline CRP levels were observed in patients who had recieved outpatient antibiotic treatment $\left(135.0 \mathrm{mg} \cdot \mathrm{L}^{-1}\right.$ versus $\left.184.0 \mathrm{mg} \cdot \mathrm{L}^{-1} ; \mathrm{p}=0.07\right)$ or outpatient treatment with inhalation steriods $\left(146.0 \mathrm{mg} \cdot \mathrm{L}^{-1}\right.$ versus $\left.185.5 \mathrm{mg} \cdot \mathrm{L}^{-1} ; \mathrm{p}=0.09\right)$. No significant association between baseline CRP levels and demographic characteristics or the presence of comorbidity was observed ( $p>0.25)$. A total of $232(80.3 \%)$ patients enrolled in the study received $\beta$-lactam monotherapy as empirical antibiotic treatment, which is a recommended initial regimen in the Netherlands for CAP patients not necessitating ICU admission and with a negative Legionella urinary antigen test, and $47(16.3 \%)$ patients received combination therapy with $\beta$-lactam and macrolide antibiotics [18]. In total, 10 (3.5\%) patients received a different empirical antibiotic therapy. Of those, four $(0.6 \%)$ patients were initially treated with doxycyclin or erythromycin monotherapy because of suspicion of an atypical cause of pneumonia on admission. In one $(0.3 \%)$ patient, combination therapy with erythromycin and rifampicin was initiated because of a strong suspicion for an L. pneumophila infection.
In $122(89.1 \%)$ out of 137 patients with established aetiology, empirical antibiotic treatment was considered appropriate. A total of $20(6.9 \%)$ patients had died by day 28 and nine $(3.1 \%)$ patients required ICU admission during follow-up (table 1).

\section{Aetiology and CRP levels}

An aetiological diagnosis could be established in 137 (47.4\%) patients. S. pneumoniae was the most frequently indentified pathogen in $55(19.0 \%)$ cases. Median (interquartile range) baseline CRP concentrations were the highest in patients with a S. pneumoniae infection (278 (147-390) $\left.\mathrm{mg} \cdot \mathrm{L}^{-1}\right)$,

\begin{tabular}{|c|c|c|}
\hline TABLE 1 & \multicolumn{2}{|c|}{$\begin{array}{l}\text { Characteristics of the study cohort of } 289 \\
\text { patients with severe community-acquired } \\
\text { pneumonia }\end{array}$} \\
\hline Age yrs & & $69.7 \pm 13.8$ \\
\hline Females & & 99 (34.3) \\
\hline PSI score & & $112.9 \pm 25.7$ \\
\hline Class IV & & $198(68.5)$ \\
\hline Class V & & $52(18.0)$ \\
\hline APACHE II s & ore & $13.8 \pm 4.6$ \\
\hline Comorbidity & & $180(62.3)$ \\
\hline Congestive & neart failure & $36(12.5)$ \\
\hline Neoplasm & & $65(22.5)$ \\
\hline Liver disea & & $3.0(1.0)$ \\
\hline Cerebrovas & ular disease & $25(8.7)$ \\
\hline Chronic rer & disease & $27(9.3)$ \\
\hline COPD & & $88(30.4)$ \\
\hline \multicolumn{3}{|c|}{ Clinical features } \\
\hline Temperatur & ${ }^{\circ} \mathrm{C}$ & $38.5 \pm 1.2$ \\
\hline Respiratory & ate breaths $\cdot \min ^{-1}$ & $26.7 \pm 8.7$ \\
\hline \multicolumn{3}{|c|}{ Laboratory data } \\
\hline $\mathrm{CRP} \mathrm{mg} \cdot \mathrm{L}^{-}$ & & $174(147-390)$ \\
\hline White bloo & cell count $10^{9} \cdot \mathrm{L}^{-1}$ & $16.5 \pm 9.2$ \\
\hline \multicolumn{3}{|c|}{ Antibiotic therapy } \\
\hline$\beta$-lactam & & $232(80.3)$ \\
\hline Amoxicill & \pm clavulanic acid & $169(58.5)$ \\
\hline Cephalos & oorin (2nd or 3rd generation) & $60(20.7)$ \\
\hline Cephtr & axone & $47(16.2)$ \\
\hline Cephts & zidime & $12(4.2)$ \\
\hline Cepho & axime & $1(0.3)$ \\
\hline Penicillin & & $3(1.0)$ \\
\hline$\beta$-lactam $/ \mathrm{m}$ & crolide combination & $47(16.3)$ \\
\hline Amoxicill & \pm clavulanic acid and macrolide & $32(11.1)$ \\
\hline Cephalos & orin (2nd or 3rd generation) and macrolide & $14(4.8)$ \\
\hline Cephtr & axone and macrolide & $11(3.8)$ \\
\hline Cephts & zidime and macrolide & $2(0.7)$ \\
\hline Other ${ }^{\#}$ & & $10(3.5)$ \\
\hline \multicolumn{3}{|l|}{ Outcome } \\
\hline ICU admiss & ons during hospitalisation & $9(3.1)$ \\
\hline 28-day mor & ality & $20(6.9)$ \\
\hline
\end{tabular}

Data are presented as mean $\pm S D, n$ (\%) or median (interquartile range). PSI: Pneunomia Severity Index; APACHE: Acute Physiology and Chronic Health Evaluation; COPD: chronic obstructive pulmonary disease; CRP: C-reactive protein; ICU: intensive care unit. " : other antibiotics include: cotrimoxazole $(n=2 ; 0.7 \%) ; \beta$-lactam and ciprofloxacin $(n=2 ; 0.7 \%)$; doxycyclin $(n=2 ; 0.7 \%)$; erytromycin and rifampicin $(n=1 ; 0.3 \%)$; levofloxacin $(n=1 ; 0.3 \%)$; and erythromycin $(n=2 ; 0.6 \%)$. 


\begin{tabular}{|c|c|c|c|c|}
\hline \multirow[t]{2}{*}{ TABLE 2} & protein & according to & & community-acquired \\
\hline & Subjects & Median CRP level & Size of range & Interquartile range \\
\hline Streptococcus pneumoniae & $55(19.0)$ & 278.0 & 686 & $147-390$ \\
\hline Haemophilus influenzae & $9(3.1)$ & 214.0 & 278 & $168-313$ \\
\hline Staphylococcus aureus & $8(2.8)$ & 187.0 & 299 & $115-330$ \\
\hline Mycoplasma pneumoniae & $5(1.7)$ & 49.0 & 299 & $27-228$ \\
\hline Legionella pneumophila $^{+}$ & $7(2.4)$ & 247.0 & 286 & $176-421$ \\
\hline Enterobacteriaciae $^{\S}$ & $15(5.2)$ & 129.0 & 452 & 53-272 \\
\hline Moraxella catarrhalis & $5(1.7)$ & 64.0 & 197 & $49-165$ \\
\hline Other pathogens ${ }^{f}$ & $11(3.8)$ & 185.0 & 403 & $117-231$ \\
\hline Multiple bacterial pathogens & $12(4.2)$ & 213.0 & 672 & $83-404$ \\
\hline
\end{tabular}

followed by L. pneumophila (247 (147-390) $\left.\mathrm{mg} \cdot \mathrm{L}^{-1}\right)$ Haemophilus influenzae $\left(214(168-313) \mathrm{mg} \cdot \mathrm{L}^{-1}\right)$, Saphylococcus aureus $\left(187(115-330) \mathrm{mg} \cdot \mathrm{L}^{-1}\right)$, Enterobacteriaciae (129.0 (53272) $\left.\mathrm{mg} \cdot \mathrm{L}^{-1}\right)$, Chlamydia pneumoniae $\left(115.5(57-317) \mathrm{mg} \cdot \mathrm{L}^{-1}\right)$, Moraxella catarrhalis $\left(64.0(49-165) \mathrm{mg} \cdot \mathrm{L}^{-1}\right)$ and $\mathrm{M}$. pneumoniae infections (49 (27-228) $\mathrm{mg} \cdot \mathrm{L}^{-1}$; table 2$)$. Patients with multiple identified bacterial pathogens had median admission CRP levels of $213.0 \mathrm{mg} \cdot \mathrm{L}^{-1}$. The aetiology of these 12 cases is specified in table 3 . The median baseline CRP levels were significantly different among the causative pathogens $(\mathrm{p}<0.01$, ANOVA). Patients with unknown aetiology had a significantly lower median CRP concentration on admission

\begin{tabular}{|c|c|c|}
\hline TABLE & $\begin{array}{l}\text { Aetiology of the } 12 \text { ce } \\
\text { pathogens specified }\end{array}$ & with multiple bacterial \\
\hline \multirow[t]{2}{*}{ Subject } & \multicolumn{2}{|c|}{ Aetiology } \\
\hline & Pathogen 1 & Pathogen 2 \\
\hline 1 & Streptococcus pneumoniae ${ }^{\#}$ & Haemophilus influenzae \\
\hline 2 & Streptococcus pneumoniae ${ }^{+}$ & Enterobacter spp \\
\hline 3 & Streptococcus pneumoniae & Eschericia coli" \\
\hline 4 & Streptococcus pneumoniae ${ }^{\#,} \bullet$ & Chlamydia pneumoniae \\
\hline 5 & Streptococcus pneumoniae ${ }^{\#,+}$ & Chlamydia pneumoniae \\
\hline 6 & Haemophilus influenzae & Chlamydia pneumoniae \\
\hline 7 & Haemophilus influenzae" & Legionella pneumophila \\
\hline 8 & Haemophilus influenzae & Staphylococcus hominis ${ }^{\#}$ \\
\hline 9 & Mycoplasma pneumoniae & Eschericia coli" \\
\hline 10 & Chlamydia pneumoniae & Staphylococcus aureus" \\
\hline 11 & Legionella pneumophila & Corynebacterium difteria ${ }^{\#}$ \\
\hline 12 & Legionella pneumophila & Streptococcus group $\mathrm{B}^{\#}$ \\
\hline
\end{tabular}

than patients with established aetiological diagnosis (140.5 mg. $\mathrm{L}^{-1}$ versus $209.0 \mathrm{mg} \cdot \mathrm{L}^{-1} ; \mathrm{p}<0.01$ ). Patients with $L$. pneumophila infection had a slower, but not statistically significant, normalisation of CRP within the first 3 days of follow-up as compared with patients with other aetiological diagnosis. The decline in CRP levels on days $0-3$ was $32.9 \%$ in patients with $L$. pneumophila infection, as compared with $38.6 \%$ in patients with pneumonia of other aetiology (mean difference $5.7 \% ; \mathrm{p}=0.58$ ). However, in the second part of the first week of follow-up, the decrease in CRP levels was larger in patients with L. pneumophila infection (48.5\%) compared with others $(28.5 \%$; mean difference 20.0; $\mathrm{p}<0.01)$.

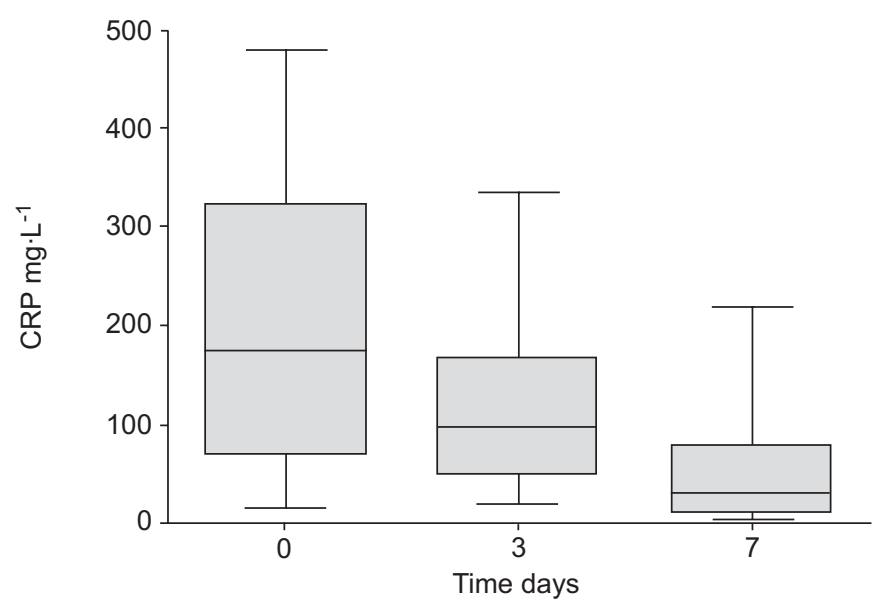

FIGURE 1. Patterns of normalisation of C-reactive protein (CRP) levels for the 289 study patients with severe community-acquired pneumonia. Horizontal lines represent the median; boxes, the interquartile range; and whiskers, the highest and lowest non-outlier values. 


\begin{tabular}{|c|c|c|c|c|c|}
\hline & Unknown aetiology & \multicolumn{4}{|c|}{ Patients with established aetiology } \\
\hline Subjects & $152(52.6)$ & $112(38.8)$ & $25(8.7)$ & & \\
\hline Day 0 & $140.5(56-293)$ & $233.0(131-358)$ & $152(63-243)$ & & \\
\hline Day 3 & $90.0(23-153)$ & $98.0(30-168)$ & $108.5(55-215)$ & & \\
\hline Day 7 & $29.0(12-79)$ & $36.0(18-75)$ & $29(15-92)$ & & \\
\hline \multicolumn{6}{|c|}{ Mean decline in CRP } \\
\hline Day 0-3 & $36.3 \pm 30.4$ & $44.5 \pm 30.5$ & $25.2 \pm 24.4$ & $19.3(6.1-32.5)$ & $<0.001$ \\
\hline Day 0-7 & $63.1 \pm 34.6$ & $75.5 \pm 24.7$ & $60.4 \pm 32.3$ & $15.1(1.8-28.5)$ & 0.03 \\
\hline
\end{tabular}

Data are presented as $\mathrm{n}(\%)$, median (interquartile range) or mean $\pm \mathrm{SD}$, unless otherwise stated. $\mathrm{Cl}$ : confidence interval. ${ }^{\#}$ : the mean difference $(95 \% \mathrm{Cl})$ in per cent decline in CRP among patients with appropriate and inappropriate antibiotic treatment (established aetiology) is displayed.

\section{The value of consecutive CRP measurements in follow-up of antibiotic treatment}

CRP measurements were performed in all patients on admission, in $264(91.3 \%)$ patients on day 3 and in 210 $(72.6 \%)$ patients on day 7 of hospitalisation. The median (range) CRP concentration was $97.5(51-163) \mathrm{mg} \cdot \mathrm{L}^{-1}$ on day 3 and $31.0(13-78) \mathrm{mg} \cdot \mathrm{L}^{-1}$ on day 7 of follow-up. Patterns of normalisation of CRP are displayed in figure 1 . The mean decline in CRP levels was 38.4\% (interquartile range 5.3-65.5\%) within the first 3 days and $80.9 \%$ (interquartile range $54.2 \%-$ $92.0 \%$ within the first week of follow-up. In univariate analysis, patients treated with inappropriate empirical antibiotics had significantly slower normalisation of CRP levels as measured in the first 3 days (mean difference 19.3\%; 95\% CI $6.1-32.5 \%$ ) and in the first week of hospitalisation (mean difference $15.1 \%$; $95 \%$ CI $1.8-28.5 \%$; table 4 ). In multivariate analysis, a decline of $<60 \%$ in CRP levels in 3 days and a decline of $<90 \%$ in CRP levels in 7 days were both associated with an increased risk of having received inappropriate empirical antibiotic treatment (day 0-3, OR 6.98, 95\% CI 1.56-31.33) and (day 0-7; OR 3.74, 95\% CI 1.12-13.77; table 5).

\begin{tabular}{|c|c|c|c|}
\hline \multirow[t]{3}{*}{ TABLE 5} & \multicolumn{3}{|c|}{$\begin{array}{l}\text { Multivariate analysis of delayed normalisation of } \\
\text { C-reactive protein (CRP) and the risk of having } \\
\text { received inappropriate antibiotic treatment }\end{array}$} \\
\hline & & \multicolumn{2}{|c|}{ Received inappropriate antibiotic treatment } \\
\hline & & OR $(95 \% \mathrm{Cl})^{\#}$ & p-value \\
\hline \multicolumn{2}{|c|}{ Day 0-3 CRP decline $<60 \%$} & $6.98(1.56-31.33)$ & 0.004 \\
\hline \multicolumn{2}{|c|}{ Day 0-7 CRP decline $<\mathbf{9 0 \%}$} & $3.74(1.12-13.77)$ & 0.04 \\
\hline
\end{tabular}

Multivariate analysis was conducted among the 137 patients with established aetiology. OR: odds ratio; $\mathrm{Cl}$ : confidence interval. ${ }^{\#}$ : the displayed ORs are adjusted for patient characteristics (age, sex and comorbid illnesses), Pneumonia Severity Index score, symptoms and signs of pneumonia (cough, sputum production, sore throat, dyspnoea, chest pain, haemoptoe, confusion, blood pressure, respiratory rate, pulse and oxygen saturation).
Patients with delayed normalisation of CRP levels in the first week had a trend towards an increased risk of mortality (OR 3.73, 95\% CI 0.46-30.52; $\mathrm{p}=0.06$ ); however when corrected for pneumonia severity, patients' charactecteristics, and symptoms or signs of pneumonia on admission, this was not statistically significant. In addition, patients with delayed normalisation of CRP in the first 3 days had a slightly increased risk of developing early or late treatment failure, but again this was not statistically significant (table 6).

\section{DISCUSSION}

The results of the present study show that consecutive measurements of CRP in follow-up of antibiotic treatment for severe CAP are useful. Delayed normalisation of CRP within the first 3-7 days of follow-up is suggestive of inappropriate empirical antibiotic therapy. Patients with a decline of $<60 \%$ in CRP levels in 3 days or a decline of $<90 \%$ in 7 days had a fourto seven-fold increased risk of having received inappropriate antibiotic treatment. Since there is limited evidence on the relevance of consecutive CRP measurements, the main findings of the present study may have clinical implications.

The results of the few previous studies concerning the usefulness of consecutive CRP measurements in follow-up of CAP are in line with the present findings [8-10]. SMITH et al. [9] studied the usefulness of CRP as marker in 28 patients who had no obvious response to treatment. They concluded that CRP could be of aid to clinicians. Another study in 53 patients with severe CAP admitted to the ICU also showed that identification of CRP patterns may be of value in follow-up of treatment [8]. Recently, MENENDEZ et al. [10] have demonstrated that a persistently high CRP level on days 1 and 3 in follow-up of patients with mild-to-severe pneumonia was independently associated with a higher risk of treatment failure. In the present cohort, patients with an inadequate decline in CRP also had a higher risk of treatment failure; however, this was not statistically significant. These different results may be explained by differences in pneumonia severity of both study populations and addressing absolute CRP values at days 1 and 3 as compared with relative changes in CRP measured on days 3 and 7 in the present study. 


\begin{tabular}{|c|c|c|c|c|c|c|c|}
\hline \multirow[t]{3}{*}{ TABLE 6} & \multicolumn{7}{|c|}{$\begin{array}{l}\text { Multivariate analysis of delayed normalisation of C-reactive protein (CRP) and the risk for having an unfavorable } \\
\text { outcome }\end{array}$} \\
\hline & & \multicolumn{2}{|c|}{ Mortality (within 28 day) } & \multicolumn{2}{|c|}{ Early (within 3 days) treatment failure } & \multicolumn{2}{|c|}{ Late (within 28 days) treatment failure } \\
\hline & & OR (95\% Cl) & p-value & OR $(95 \% \mathrm{Cl})$ & p-value & OR $(95 \% \mathrm{Cl})$ & $\mathrm{p}$-value \\
\hline \multirow{2}{*}{\multicolumn{2}{|c|}{$\begin{array}{l}\text { Day 0-3 CRP decline }<60 \% \\
\text { Dav 0-7 CRP decline }<90 \%\end{array}$}} & $1.09(0.32-3.73)$ & 0.89 & $1.57(0.85-2.92)$ & 0.16 & $1.29(0.62-2.68)$ & 0.50 \\
\hline & & $1.23(0.45-2.99)$ & 1.00 & & & $0.87(0.39-1.94)$ & 0.74 \\
\hline
\end{tabular}

The displayed odds ratios (ORs) are adjusted for patient characteristics (age, sex and comorbid illnesses), Pneumonia Severity Index score, symptoms and signs of pneumonia (cough, sputum production, sore throat, dyspnoea, chest pain, hemoptoe, confusion, blood pressure, respiratory rate, pulse and oxygen saturation) Cl: confidence interval.

In univariable assessment, a delayed decline in CRP levels was associated with a trend towards increased risk for mortality. However, after correction for pneumonia severity, patients' characteristics and clinical variables at baseline, a statistically significant relationship could not be established. A recent study has shown that failure of CRP to decrease leads to an increased risk of mortality [21].

Interestingly, baseline CRP levels appeared to be influenced by the causative pathogen, antibiotic use prior to hospitalisation and the use of inhalation steroids. Theoretically, baseline CRP levels could be of use in determining aetiology of severe CAP, but the ability of CRP to differentiate in aetiology of severe CAP is low [22]. As indicated by the results of the present study and others, CRP levels are influenced by the use of steroids. Moreover, it has been reported that treatment with steroids leads to suppression of CRP production [23]. A study by PERREN et al. [24] demonstrated that corticosteroids did not influence the time-dependent decline of CRP levels. However, according to the present results, the use of steroids needs to be considered in order to interpret CRP levels in follow-up correctly. Concerning the influence of the causative pathogen on the decay of CRP, a slower decline in CRP levels was observed in the first 3 days of follow-up in L. pneumophila than in other pathogens. This may be due to inappropriate empirical treatment; however, all patients had Legionella antigen test performed within $12 \mathrm{~h}$ and patients with a positive Legionella antigen test received treatment for Legionella infection within $12 \mathrm{~h}$. Another explanation may be that L. pneumophila, as an intracellular pathogen, causes a different host response to infection, characterised by prolonged and greater increases of CRP $[25,26]$. According to these results, the causative pathogens need to be taken into account in order to interpret CRP levels in follow-up correctly. For example, in case of an established L. pneumophila infection, persistent high CRP levels should not be the sole reason for antibiotic switch or additional invasive diagnostic procedures. The results of the present study indicate that a delayed decline in CRP levels is related to inappropriate empirical antibiotic treatment. Conversely, CRP levels returning to normal ranges might indicate that duration of antibiotic treatment has been sufficient, allowing earlier discontinuation of antibiotics or a switch to oral antibiotics. Such a CRP-based management strategy could potentially help in reducing antibiotic usage, costs, toxicity, length of hospital stay and the risk of emerging resistance [8]. However, this concept needs to be addressed in further studies.
The present study has three important limitations. First, focus was placed on episodes of severe CAP in patients without the primary need for ICU admission. Because acute phase proteins, such as CRP, reflect the intensity of inflammation, generalisibility to patients with less severe pneumonia can be questioned [27]. Secondly, appropriate treatment was defined as "at least one antibiotic covering all of the causative pathogens identified". However, the causative role in CAP can be debated for some of the isolated bacteria. When these isolates only represent colonisation of the respiratory tract, the association of a delayed decline and the risk of having received inappropriate therapy may be overestimated. Thirdly, daily CRP measurements could have added more information to the study. However, the study was designed in accordance with a previous study taking clinically relevant time-points after admission [21].

In conclusion, consecutive C-reactive protein measurements are useful in the first week in follow-up of antibiotic treatment for severe community-acquired pneumonia, when taking the causative microorganism and use of steroids into account. A delayed decline in C-reactive protein levels is associated with a higher risk of having received inappropriate antibiotic treatment.

\section{REFERENCES}

1 Fry AM, Shay DK, Holman RC, Curns AT, Anderson LJ. Trends in hospitalizations for pneumonia among persons aged 65 years or older in the United States, 1988-2002. JAMA 2005; 294: 2712-2719.

2 Mandell LA, Wunderink RG, Anzueto A, et al. Infectious Diseases Society of America/American Thoracic Society consensus guidelines on the management of communityacquired pneumonia in adults. Clin Infect Dis 2007; 44: Suppl. 2, S27-S72.

3 Woodhead M, Blasi F, Ewig S, et al. Guidelines for the management of adult lower respiratory tract infections. Eur Respir J 2005; 26: 1138-1180.

4 Colice GL, Morley MA, Asche C, Birnbaum HG. Treatment costs of community-acquired pneumonia in an employed population. Chest 2004; 125: 2140-2145.

5 Malhotra-Kumar S, Lammens C, Coenen S, Van HK, Goossens $\mathrm{H}$. Effect of azithromycin and clarithromycin therapy on pharyngeal carriage of macrolide-resistant streptococci in healthy volunteers: a randomised, doubleblind, placebo-controlled study. Lancet 2007; 369: 482-490. 
6 Couto RC, Barbosa JA, Pedrosa TM, Biscione FM. C-reactive protein-guided approach may shorten length of antimicrobial treatment of culture-proven late-onset sepsis: an intervention study. Braz J Infect Dis 2007; 11: 240-245.

7 Clyne B, Olshaker JS. The C-reactive protein. J Emerg Med 1999; 17: 1019-1025.

8 Coelho L, Povoa P, Almeida E, et al. Usefulness of Creactive protein in monitoring severe community-acquired pneumonia clinical course. Crit Care 2007; 11: R92.

9 Smith RP, Lipworth BJ, Cree IA, Spiers EM, Winter JH. C-reactive protein. A clinical marker in communityacquired pneumonia. Chest 1995; 108: 1288-1291.

10 Menendez R, Cavalcanti M, Reyes S, et al. Markers of treatment failure in hospitalized community-acquired Pneumonia. Thorax 2008; 63: 447-452.

11 Prat C, Dominguez J, Andreo F, et al. Procalcitonin and neopterin correlation with etiology and severity of pneumonia. J Infect 2006; 52: 169-177.

12 Oosterheert JJ, Bonten MJ, Schneider MM, et al. Effectiveness of early switch from intravenous to oral antibiotics in severe community acquired pneumonia: multicentre randomised trial. BMJ 2006; 333: 1193-1197.

13 Niederman MS, Mandell LA, Anzueto A, et al. Guidelines for the management of adults with community-acquired pneumonia. Diagnosis, assessment of severity, antimicrobial therapy, and prevention. Am J Respir Crit Care Med 2001; 163: 1730-1754.

14 Fine MJ, Auble TE, Yealy DM, et al. A prediction rule to identify low-risk patients with community-acquired pneumonia. N Engl J Med 1997; 336: 243-250.

15 Knaus WA, Draper EA, Wagner DP, Zimmerman JE. APACHE II: a severity of disease classification system. Crit Care Med 1985; 13: 818-829.

16 Jacobs E. Serological diagnosis of Mycoplasma pneumoniae infections: a critical review of current procedures. Clin Infect Dis 1993; 17: Suppl. 1, S79-S82.
17 Stout JE, Yu VL. Legionellosis. N Engl J Med 1997; 337: 682-687.

18 Schouten JA. Revised SWAB guidelines for antimicrobial therapy of community acquired pneumonia. Amsterdam, SWAB, 2005. Available from www.swab.nl.

19 Halm EA, Fine MJ, Marrie TJ, et al. Time to clinical stability in patients hospitalized with community-acquired pneumonia: implications for practice guidelines. JAMA 1998; 279: 1452-1457.

20 Chow AW, Hall CB, Klein JO, Kammer RB, Meyer RD, Remington JS. Evaluation of new anti-infective drugs for the treatment of respiratory tract infections. Infectious Diseases Society of America and the Food and Drug Administration. Clin Infect Dis 1992; 15: Suppl. 1, S62-S88.

21 Chalmers JD, Singanayagam A, Hill AT. C-reactive protein is an independent predictor of severity in communityacquired pneumonia. Am J Med 2008; 121: 219-225.

22 van der Meer V, Neven AK, van den Broek PJ, Assendelft WJ. Diagnostic value of $\mathrm{C}$ reactive protein in infections of the lower respiratory tract: systematic review. BMJ 2005; 331: 26-32.

23 Man SF, Sin DD. Effects of corticosteroids on systemic inflammation in chronic obstructive pulmonary disease. Proc Am Thorac Soc 2005; 2: 78-82.

24 Perren A, Cerutti B, Lepori M, et al. Influence of steroids on procalcitonin and C-reactive protein in patients with COPD and community-acquired pneumonia. Infection 2008; 36: 163-166.

25 Holmberg H, Bodin L, Jonsson I, Krook A. Rapid etiological diagnosis of pneumonia based on routine laboratory features. Scand J Infect Dis 1990; 22: 537-545.

26 Garcia VE, Martinez JA, Mensa J, Sanchez F, Marcos MA, de Roux A, Torres A. C-reactive protein levels in community-acquired pneumonia. Eur Respir J 2003; 21: 702-705.

27 Gabay C, Kushner I. Acute-phase proteins and other systemic responses to inflammation. N Engl J Med 1999; 340: 448-454. 